\title{
Emisión de gases de efecto invernadero en dos sitios de disposición final de residuos sólidos urbanos en México
}

\section{Greenhouse Gases Emission from two Disposal Sites of Municipal Solid Wastes in Mexico}

Díaz-Archundia Laura Verónica

Instituto Tecnológico de Toluca, Metepec, Edo. de México

División de Estudios de Posgrado e Investigación

Laboratorio de Investigación en Ingeniería Ambiental

Correo: lauradiazarch@yahoo.com.mx

Buenrostro-Delgado Otoniel

Universidad Michoacana de San Nicolás de Hidalgo, Morelia, Michoacán

Instituto de Investigaciones Agropecuarias y Forestales

Laboratorio de Residuos Sólidos y Medio Ambiente

Correo: otonielb@umich.mx

Mañón-Salas María del Consuelo

Instituto Tecnológico de Toluca, Metepec, Edo. de México

División de Estudios de Posgrado e Investigación

Laboratorio de Investigación en Ingeniería Ambiental

Correo: consuelomanon@gmail.com

Hernández-Berriel María del Consuelo

Instituto Tecnológico de Toluca, Metepec, Edo. de México

División de Estudios de Posgrado e Investigación

Laboratorio de Investigación en Ingeniería Ambiental

Correo:mhernandezb@toluca.tecnm.mx

\section{Resumen}

En México, 74\% de los Residuos Sólidos Urbanos (RSU) generados se disponen en rellenos sanitarios (RESA), sin embargo, los reportes sobre las condiciones de operación de estos, así como de los tiraderos a cielo abierto (TCA) son escasos. El objetivo del estudio fue determinar la generación del metano como Gas de Efecto Invernadero en un RESA y un TCA. Para ello, se evaluaron las condiciones de operación, se caracterizaron los RSU confinados en temporadas de lluvias y estiaje y se determinó la generación de metano puntual y difusa. Con el fin de mejorar la operación del RESA bajo estudio, se detectaron ocho áreas de oportunidad y se documentaron los problemas ambientales del TCA. Si bien, el tipo de operación y la cantidad de materia biodegradable en los RSU (50.36\% en RESA y 58.49\% en TCA) influencian directamente la generación de metano por tonelada de RSU, en el TCA se incrementa en temporada de lluvias, mientras que en los pozos de venteo del RESA fue 15\% más alto en temporada de estiaje, a causa de la recirculación de lixiviados durante la misma. Las emisiones superficiales de metano en el TCA fueron $97 \%$ más bajas que en el RESA, debido a su posible dispersión en los alrededores, lo cual es un riesgo potencial que requiere atención inmediata de las autoridades.

Descriptores: sitio de disposición final, relleno sanitario, tiradero a cielo abierto, metano, residuos sólidos urbanos. 


\begin{abstract}
In Mexico, $74 \%$ of the Municipal Solid Waste (MSW) produced are disposed in Landfills, however the operating conditions reported of the landfills as well as the open dumps sites are scarce the open dumps sites (ODS). The objective of this study was to determine the methane generation as Greenhouse gas in a Landfill and in an ODS. The operating conditions of the disposal sites were evaluated, the MSW disposed were characterized in the wet (rainy) and dry (Dec-May) seasons, and the methane generation in venting wells and the surface emissions were determinated. In order to improve the disposal conditions of the study site operations, eight opportunity areas were detected and environmental problems of the ODS were documented. Even though, the type of sites operation and the amount of biodegradable matter in the MSW (50.36\% in Landfill and 58.49\% in ODS) influence directly the methane generation per ton of MSW, in ODS is increased during the wet season, while venting wells Landfill was 15\% higher in the dry season due to leachate recirculation. The surface methane emissions in the ODS were 97\% lower than in the Landfill, due to a possible spread trough the pores of soil which is a potential risk that requires immediate attention from the authorities.
\end{abstract}

Keywords: final disposal site, landfill, open dump site, methane, wastes.

\section{INTRODUCCIÓN}

América Latina y el Caribe en 2010 reportaron 588.65 millones de habitantes y una generación de RSU de 0.93 $\mathrm{kg} / \mathrm{hab}$-día, de los cuales solo $54.4 \%$ llegó a sitios de disposición final (SDF) (IERMRSUALC, 2010). La población de México ascendió a 119.5 millones de habitantes en 2015 , con un crecimiento poblacional de $1.4 \%$, lo que condujo al aumento en la demanda de bienes y servicios, así como al incremento en la generación de RSU (INEGI, 2016). Estos RSU requieren de una gestión apropiada, ya que el pasivo ambiental por su confinamiento inadecuado en el país es alto. Esta disposición final se realiza tradicionalmente en RESA, donde se llega a la estabilización con la degradación de los residuos. El proceso de degradación genera contaminantes como lixiviados y biogás, ambos influenciados por la composición de los RSU y la operación del sitio, contribuyendo el biogás al Calentamiento Global, debido a que contiene principalmente $\mathrm{CH}_{4}$ y $\mathrm{CO}_{2}$, ambos gases de efecto invernadero (GEI). Conforme a lo anterior y debido a que se carece de información confiable en la mayoría de los SDF del país, el objetivo de este estudio fue determinar la generación del $\mathrm{CH}_{4}$ en un RESA y en un TCA, con el fin de servir y motivar la revisión de la operación de sitios, la caracterización de sus RSU y la influencia de estos en la generación de $\mathrm{CH}_{4}$.

\section{FundAMENTOS}

LA GeStión de RESIDUOS SÓlIDOS URBANOS EN MÉXICO

La falta de financiamiento e infraestructura principalmente en los municipios pequeños, es uno de los problemas que cada gobierno debe enfrentar con el fin de alcanzar una adecuada disposición de los RSU. Estos municipios, en el mejor de los casos, deben depositar sus residuos en los RESA de las ciudades cercanas, asumien- do los gastos de transporte y cuotas por disponer en sitios ajenos. En el peor de los casos, terminan dejando sus RSU en TCA, provocando con esto contaminación en aire, agua y suelo. México en el año 2012, generó 42.10 millones de toneladas de RSU que fueron dispuestos en 1883 SDF, de los cuales solo 238 eran RESA. Del total generado se confinó $74.39 \%$ en RESA y rellenos de tierra controlados, $20.62 \%$ en TAC y solo $4.99 \%$ se recicló (OCDE-1, 2014a). Es preocupante que en México el número de los tres tipos de sitios de disposición de 2000 a 2012 se haya triplicado, alcanzando 1883, de los cuales 1645 fueron TCA (SEMARNAT-INECC, 2012).

Durante la operación de los SDF se deben implementar revisiones periódicas y supervisiones de operación. En México, la PROFEPA y la Secretaría del Medio Ambiente y Recursos Naturales (SEMARNAT) vigilan los RESA conforme a los lineamientos establecidos en la normatividad Federal, Estatal y Local (Guía de Cumplimiento de la NOM-083-SEMARNAT-2003, SEMARNAT-1, 2016), que se basa también en estándares internacionales. La PROFEPA solicita el monitoreo constante de los parámetros fisicoquímicos de los lixiviados y del biogás, además recomienda que los RSU se caractericen al menos una vez al año para determinar el contenido de carbono que puede transformarse en $\mathrm{CH}_{4} \mathrm{y}$ $\mathrm{CO}_{2}$, con lo que los gobiernos municipales podrían estimar sus emisiones y tener un mayor control sobre ellos (SEMARNAT-1, 2016). Sin embargo, es difícil tener acceso a la información y en muchos casos esta no se encuentra actualizada, además de que para los TCA se carece de datos confiables o no hay registros.

\section{Emisiones de Gases de Efecto Invernadero}

De acuerdo con la Organización para la Cooperación y Desarrollo Económicos (OCDE) la contribución mundial de las emisiones de GEI de todos los sectores entre los años 1990 y 2000, incluyendo residuos sólidos, fue de 
20,973 Megatoneladas equivalentes de $\mathrm{CO}_{2}\left(\mathrm{MtCO}_{2} \mathrm{e}\right)$ y de 30,523 $\mathrm{MtCO}_{2}$ e del 2000 a 2010 (CICC, 2006; OCDE, 2014b). En México, durante el año 2010, las emisiones de GEI fueron de $748.252 \mathrm{MtCO}_{2} \mathrm{e}$ con un incremento de $33.4 \%$ en los últimos 20 años. Los sectores responsables de las emisiones fueron: Sector energético (67.3\%), Procesos Industriales (8.2\%), Cambio de Uso de Suelo y Silvicultura (6.3\%), Agricultura (12.3\%) y Residuos (5.9\%). Este último sector incluye la descomposición de RSU en RESA y plantas de tratamiento de aguas residuales (INECC, 2012). El INE ha reportado que México podría reducir hasta $30 \%$ sus emisiones en 2020 y 53\% en 2030 y, de forma particular en el sector de residuos, disminuir hasta $20 \%$ en 2020 y $41 \%$ en 2030 , con estrategias como el saneamiento de TCA y el aprovechamiento energético de biogás en los RESA para la generación de energía eléctrica y calórica (INE, 2010). Otra opción para reducir las emisiones de $\mathrm{CH}_{4}$ es disminuir la fracción orgánica (FO) en los RSU, para lo cual es necesario implementar programas de separación y valorización, así como incrementar la recuperación de reciclables y realizar caracterizaciones periódicas en los sitios (Hyung et al., 2013).

Fuentes de GENERACIÓN DE $\mathrm{CH}_{4}$ EN LOS SITIOS DE DISPOSICIÓN

La biodegradación de los RSU en un RESA y su generación del biogás (metanización) tiene que ver con la coordinación entre bacterias que depende, a su vez, de los microambientes (Czepiel et al., 2003; Jokela et al., 2002; Madigan et al., 2004). Estos microambientes a nivel macro se identifican como factores ambientales y operacionales (Zhang et al., 2008). Entre los ambientales se tienen la cantidad, características y descomposición de los RSU (constituyentes orgánicos e inorgánicos) y el clima (temperatura, precipitación pluvial, etcétera); mientras que los factores operacionales incluyen la compactación, tiempo de disposición (año de inicio y clausura), trituración, material de cobertura intermedia y final (características del suelo y altura), recirculación del lixiviado (humidificación) (GMI, 2012), cantidad de RSU dispuestas en una celda y su profundidad (Chen et al., 2008; Wrefor et al., 2000).

La generación de biogás comienza por lo general alrededor de los 6 a 8 meses de colocados los RSU y se prolonga por un lapso de 50 años después de la clausura del sitio. Aunque, es posible detectar $\mathrm{CH}_{4}$ (a bajas concentraciones) al inicio de la biodegradación, es en la fase de fermentación acelerada donde las concentraciones de $\mathrm{CH}_{4}$ se incrementan hasta valores entre 50 y $70 \%$ volumen (\%v/v) (Benson et al., 2007). Las emisiones de $\mathrm{CH}_{4}$ en un SDF se pueden presentar a partir de pozos de venteo, superficie y taludes con altas variabilidades espacial y temporal. En diversas partes del mundo se han realizado estudios sobre la producción de biogás a partir de puntos establecidos o medibles en una Celda, es decir, los pozos de venteo en los que se delimitan los factores que influyen en dicha generación (López, 2010; Mohammed et al, 2009; Wang et al, 2006); sin embargo, solo algunos miden las emisiones en los taludes y en la superficie de la Celda. A este tipo de emisiones se les llama emisiones difusas y contribuyen con un porcentaje considerable (30 a 60\%) de las emisiones totales de un sitio (Czepiel et al., 2003; Barry et al., 2004; Spokas et al., 2003).

Cabe mencionar que los trabajos sobre emisiones difusas en RESA o en TCA son escasos (ADEME, 2008; Babilotte et al., 2010). Para el caso de México, resulta preocupante que la información sea incompleta o en algunos casos el procesamiento de los datos sea lento, debido a que afecta la elaboración del Reporte Nacional de Emisiones retardando su publicación.

\section{Método}

\section{DESCRIPCIÓN DE SITIOS DE ESTUDIO}

Los sitios de estudio se encuentran en el Estado de México, ubicado en el centro del país (figura 1). El RESA se localiza cerca de una zona industrial y tiene una altitud de 2772 msnm, con clima templado-subhúmedo (Cwb), una temperatura media anual de $12.0^{\circ} \mathrm{C}$ y una precipitación de 1225.6 mm. El RESA tiene un área total de 95,918 $\mathrm{m}^{2}$ en la que se distribuyen una Celda saneada y cinco celdas que cumplen con la NOM-083-SEMARNAT-2003 (SEMARNAT-1, 2016) y tienen instalados 25 pozos (figura 2). Las Celdas 1, 2 y 3 están clausuradas, la Celda 4 se encuentra en operación y la celda 5 en construcción. El RESA cuenta con dos fosas de lixiviados.

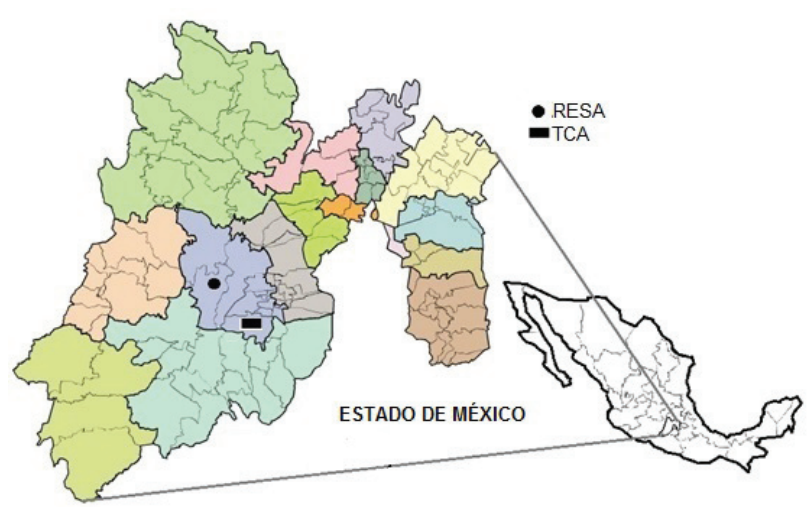

Figura 1. Ubicación del RESA y el TCA, Estado de México 
El TCA se ubica en una zona agrícola y comercial y tiene una altitud de $2600 \mathrm{msnm}$ (figura 1). El clima es templado subhúmedo (Cwb), su temperatura media es de $13.5^{\circ}$ y precipitación anual de $852.9 \mathrm{~mm}$. El TCA cuenta con una celda saneada clausurada a un lado, que no se considera para este estudio (figura 2). El TCA en operación tiene un pozo de venteo y una fosa de lixiviados. La operación de los sitios se evaluó mediante la aplicación de una lista de verificación (LV), que fue elaborada con base en los criterios de la norma NOM-083-SEMARNAT-2003 (SEMARNAT-1, 2016). Esta lista consta de siete apartados donde cada uno de ellos contiene varias preguntas. La LV se aplicó con ayuda de los responsables de seguridad y operación de cada uno de los sitios.
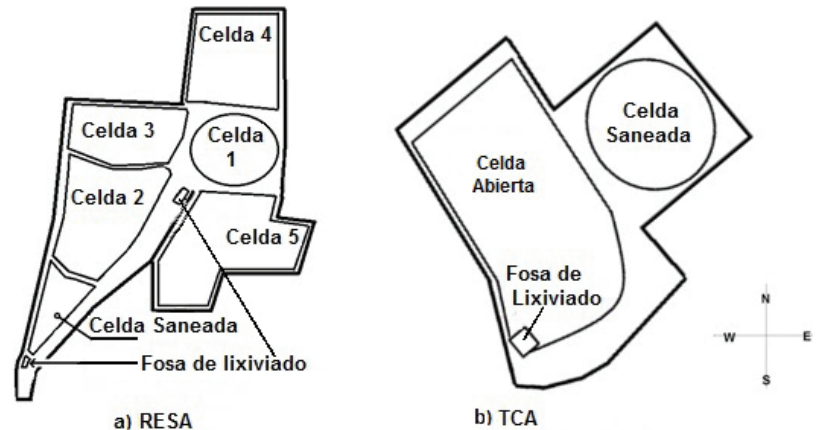

Figura 2. Sitios de disposición final de RSU del Estado de México, a) RESA, b) TCA

\section{CARACTERIZACIÓN DE RSU}

En el caso del RESA se eligieron las Celdas 1 y 3 para caracterizar los RSU. Se seleccionaron aleatoriamente cinco puntos en cada una de ellas con el fin de cubrir el total del área. Para el TCA se siguió el procedimiento descrito anteriormente en la celda en operación. En cada sitio se realizaron muestreos durante un año. Los RSU de los cinco puntos se depositaron en un solo lugar y se mezclaron para conformar una muestra compuesta de cada celda y mediante el método de cuarteo (NMX-AA-015-1985) se obtuvieron muestras mayores a $50 \mathrm{~kg}$. Estas muestras se caracterizaron de acuerdo con la norma mexicana NMX-AA-022-1985. Se pesó cada subproducto y se fraccionó para formar una muestra representativa, que se analizó fisicoquímicamente. Los análisis efectuados a cada una de las muestra representativas fueron: $\mathrm{pH}$ (NMX-AA-013-SCFI-2006), \%Hbh (NMX-AA-16-1984), SVT (Método 2540G), MO (NMXAA-021-1985) y relación Carbono/Nitrógeno (C/N) (NMX-AA-067-1985) (APHA, 2005; SEMARNAT-1, 2016). Posteriormente, los subproductos clasificados se agruparon con base en las categorías de degradación sugeridas por el Modelo Mexicano de Biogás, con el fin de identificar los diferentes grados de biodegradación para estos (SCS, 2009).

MUESTREO Y CUANTIFICACIÓN DE $\mathrm{CH}_{4}$

Para el RESA se muestrearon 24 pozos de venteo de biogás y las superficies de las Celdas 1, 2 y 3 . En el TCA se analizó el único pozo activo, ya que los otros pozos fueron destruidos. Para el muestreo en superficie se utilizaron cámaras estáticas de 15.08 L que se colocaron en puntos seleccionados sobre la capa superficial de cada celda. La selección espacial de puntos se realizó con base en la metodología de la Enviromental Agency (EA) del Reino Unido, distribuyéndolos de un modo aleatorio simple considerando la distancia recomendada de $30 \mathrm{~m}$ entre punto y punto (EA, 2009; Zhang et al., 2013). El muestreo y análisis del biogás se realizó en los pozos de venteo y en la superficie mediante un medidor de gases portátil GEM Modelo 2000 LANDTEC®. El análisis de biogás en pozos fue de $40 \mathrm{~s}$, tiempo recomendado por el proveedor del equipo GEM portátil (LANDTEC, 2009). Las lecturas en superficie se realizaron a diferentes tiempos, iniciando la primera lectura (tiempo cero) al momento de ser instalada la cámara, las siguientes lecturas fueron cada $30 \mathrm{~min}$ hasta que se detectó la estabilización de la concentración de $\mathrm{CH}_{4}$.

La generación de $\mathrm{CH}_{4}$ en pozos representada por $\mathrm{F}_{\mathrm{CH}_{4} \text { pozo }}\left(\mathrm{g} / \mathrm{m}^{2} . \mathrm{h}\right)$ se calculó por medio de las ecuaciones 1 y 2, donde los moles de $\mathrm{CH}_{4}$ representadas por $n_{\mathrm{CH}_{4}}$ se estimaron con la ecuación 1, a partir de la información de cada pozo obtenida por medio del GEM portátil, donde $P_{\mathrm{CH}_{4}}$ corresponde a la presión (atm), $F_{\mathrm{CH}_{4}}$ es el flujo de $\mathrm{CH}_{4}\left(\mathrm{~m}^{3} / \mathrm{h}\right), R$ corresponde a la constante de los gases $\left(\mathrm{atm} \cdot \mathrm{m}^{3} / \mathrm{K} \cdot \mathrm{mol}\right)$ ) y $T$ es la temperatura (K). En la ecuación 2, $\mathrm{PM}_{\mathrm{CH}_{4}}$ corresponde al Peso Molecular del $\mathrm{CH}_{4}(\mathrm{~g} / \mathrm{mol})$, $A$ es el área del pozo $\left(\mathrm{m}^{2}\right)$ y $n_{\mathrm{CH}_{4}}$ son las moles del $\mathrm{CH}_{4}(\mathrm{~mol})$ calculadas con la ec. 1 .

$n_{\mathrm{CH}_{4}}=P_{\mathrm{CH}_{4}}{ }^{*} F_{\mathrm{CH}_{4}} / R^{*} \mathrm{~T}$

$F_{\mathrm{CH}_{4} \text { pozo }}=n_{\mathrm{CH}_{4}}{ }^{*} \mathrm{PM}_{\mathrm{CH}_{4}} / \mathrm{A}$

La generación de $\mathrm{CH}_{4}$ en superficie representada por $F_{\mathrm{CH}_{4} \text { sup }}\left(\mathrm{g} / \mathrm{m}^{2} . \mathrm{h}\right)$ se calculó por medio de las ecuaciones 3 y 4. Primero se calculó con la ecuación 3 el volumen de $\mathrm{CH}_{4}$ simbolizado por $V_{\mathrm{CH}_{4}}(\mathrm{~L})$, donde $V_{\text {camp }}$ es el volumen de la campana (L) y $Y_{\mathrm{CH}_{4}}$ es la fracción de $\mathrm{CH}_{4}$ en el biogás. Con el volumen calculado se obtuvo el número de moles del $\mathrm{CH}_{4}$ a partir de la ecuación de los gases ideales, convirtiendo moles a gramos mediante el peso molecular $\mathrm{CH}_{4}$, para posteriormente calcular el $F_{\mathrm{CH}_{4} \text { sup }}$ con la ecuación 4 , donde $\Delta_{\text {masa }}(\mathrm{g})$ es la diferencia de la masa fi- 
nal e inicial del $\mathrm{CH}_{4}$ acumulado en la campana, $\Delta_{t}$ es la diferencia de tiempo entre el inicio y la estabilización de la lectura (h) y $A_{\text {camp }}$ es el área de la campana $\left(\mathrm{m}^{2}\right)$.

$$
\begin{aligned}
& V_{\mathrm{CH}_{4}}=V_{\text {camp }}{ }^{*} Y_{\mathrm{CH}_{4}} \\
& F_{\mathrm{CH}_{4} \text { sup }}=\Delta_{\text {masa }} / \Delta t^{*} A_{\text {camp }}
\end{aligned}
$$

\section{RESULTADOS Y DISCUSIÓN}

\section{OPERACIÓN DE LOS SITIOS DE DISPOSICIÓN FINAL}

El concentrado de los resultados obtenidos de la aplicación de la LV y sus observaciones se presentan en la tabla 1. El RESA empezó a recibir RSU desde 1989 operando inicialmente como un TCA. En 2003 una empresa privada comenzó las actividades de saneamiento, recuperación y regularización del sitio. Una vez saneado, se construyeron celdas conforme a la norma NOM-
083-SEMARNAT-2003, operándose la celda 1 de 2008 a 2009, la celda 2 de 2010 a 2011 y la celda 3 de 2012 a 2014.

El RESA recibe en promedio 650 toneladas por día (t/d) de RSU, los cuales se vierten en el frente de tiro, donde los segregadores primarios (pepenadores) separan los materiales susceptibles de recuperación (cartón, PET, metal, vidrio). A continuación, los RSU se compactan a $830 \mathrm{~kg} / \mathrm{m}^{3}$ mediante un equipo marca CAT, modelo $816 \mathrm{~F}$. Las celdas tipo RESA (celdas 1-5) tienen un recubrimiento inferior que se constituye por tres capas impermeables, la primera se compone por material arcilloso con un espesor de $0.30 \mathrm{~m}$ compactado a $90 \%$ de la prueba Proctor, la segunda consiste de una geomembrana de polietileno de alta densidad de $1.5 \mathrm{~mm}$ de espesor y la tercera capa sirve de protección a la geomembrana y está compuesta por $0.20 \mathrm{~m}$ de material arcilloso compactado a $85 \%$ de la prueba Proctor (SEMARNAT-1, 2009; EPA, 2000).

\begin{tabular}{|c|c|c|c|c|}
\hline Sitio & RESA & Áreas de oportunidad & TCA & Áreas de oportunidad \\
\hline Categoría & $\mathrm{A}$ & & $\mathrm{C}$ & \\
\hline $\begin{array}{l}\text { Proyecto ejecutivo y } \\
\text { evaluación }\end{array}$ & $100 \%$ & No encontradas & $0 \%$ & $\begin{array}{c}\text { No se cuenta con proyecto } \\
\text { ejecutivo y evaluación de impacto } \\
\text { ambiental }\end{array}$ \\
\hline $\begin{array}{l}\text { Restricción para ubicación } \\
\text { del sitio }\end{array}$ & $100 \%$ & No encontradas & $0 \%$ & $\begin{array}{c}\text { No se tiene estudio previo de la } \\
\text { ubicación, se encuentra en una } \\
\text { área protegida, distancia mínima a } \\
\text { un desarrollo urbano }\end{array}$ \\
\hline $\begin{array}{l}\text { Estudios y análisis previos } \\
\text { para la selección del sitio }\end{array}$ & $100 \%$ & No encontradas & $0 \%$ & $\begin{array}{c}\text { No se cuenta con estudio } \\
\text { geológico (fallas, fracturas) e } \\
\text { hidrológico }\end{array}$ \\
\hline $\begin{array}{l}\text { Estudios y análisis previos } \\
\text { en el sitio }\end{array}$ & $90 \%$ & $\begin{array}{l}\text { No se determinaron inicialmente } \\
\text { características estratigráficas }\end{array}$ & $0 \%$ & $\begin{array}{l}\text { No se cuenta con estudios } \\
\text { geológicos e hidrogeológicos }\end{array}$ \\
\hline $\begin{array}{l}\text { Estudios de generación y } \\
\text { composición }\end{array}$ & $80 \%$ & $\begin{array}{c}\text { No se realiza balance hídrico del } \\
\text { lixiviado }\end{array}$ & $0 \%$ & $\begin{array}{c}\text { No realizan estudios de } \\
\text { generación y composición de RSU, } \\
\text { lixiviados y biogás }\end{array}$ \\
\hline $\begin{array}{c}\text { Características } \\
\text { constructivas y operativas } \\
\text { del sitio }\end{array}$ & $70 \%$ & $\begin{array}{l}\text { No garantiza la extracción del biogás, } \\
\text { no cuenta con área de emergencia, } \\
\text { cuenta con programa de control de } \\
\text { fauna nociva pero no se controla, los } \\
\text { residuos se cubren cada que se alcanza } \\
\text { la altura de la terraza, no se conoce el } \\
\text { grado de estabilización de los residuos }\end{array}$ & $20 \%$ & $\begin{array}{l}\text { No garantiza la extracción del } \\
\text { biogás, no realiza la quema } \\
\text { del biogás, no cuenta con área } \\
\text { de emergencia en casos de } \\
\text { eventualidad, no controla la } \\
\text { fauna nociva, los residuos no se } \\
\text { cubren y no se conoce el grado de } \\
\text { estabilización de los residuos }\end{array}$ \\
\hline Clausura del sitio & $95 \%$ & $\begin{array}{c}\text { No cuenta aún con programa de } \\
\text { monitoreo posclausura }\end{array}$ & $0 \%$ & $\begin{array}{l}\text { No cuenta con programa de } \\
\text { monitoreo posclausura y plan de } \\
\text { uso posclausura para el sitio }\end{array}$ \\
\hline Cumplimiento Total \% & $91 \%$ & & $3 \%$ & \\
\hline
\end{tabular}

Tabla 1. Evaluación de condiciones de operación conforme a la LV para el RESA y el TCA 
A la celda en operación eventualmente se le coloca cobertura diaria y la cobertura intermedia se instala una vez que la terraza alcanza una altura promedio de $7 \mathrm{~m}$, altura dentro del rango recomendado de 4-10 $\mathrm{m}$ por la International Solid Waste Association (ISWA) (GMI, 2012). Los materiales utilizados en la base y superficie de la celda (corona y taludes) son del mismo tipo de suelo del sitio (tobas arcillosas y arcillo-arenosas), los cuales ayudan a prevenir infiltraciones, la dispersión de los RSU, la generación de malos olores y la fauna nociva. Para el manejo de lixiviados se cuenta con cárcamos y drenes colectores que los dirigen a las dos fosas de lixiviados (figura 2).

En el RESA se recirculan lixiviados una vez por semana con una pipa de $8000 \mathrm{~L}$, los cuales se vierten en coronas, taludes y caminos de acuerdo con la capacidad existente en las fosas y a las necesidades propias de humidificación. Los pozos de venteo son tubos de PVC rodeados con una estructura de piedras en la parte superior, para que a su vez, el biogás se queme o utilice en un futuro para la generación de energía eléctrica. La ubicación de los pozos tiene un radio de influencia de $25 \mathrm{~m}$. Conforme a la evaluación realizada, el RESA alcanzó una puntuación promedio de 91\%, destacando como áreas de oportunidad las categorías de estudios previos al sitio, estudios de generación y composición y, características constructivas y operativas del sitio.

En cuanto al TCA, inició operaciones alrededor del año 1980 (comentario personal del encargado del sitio). Durante este año el TCA se cercó con malla ciclónica, que fue destruida por falta de seguridad, posteriormente ocurrieron conatos accidentales de incendios y la destrucción de los pozos de venteo. La Celda Saneada a un lado de la Celda abierta actualmente no tiene pozos y está cubierta por montículos de tierra de teyolote de una mina ubicada a un costado del sitio. La Celda abierta ubicada al sur-poniente del TCA inició su operación en 1995 y finalizó en enero del 2014. Actualmente solo existe un pozo de venteo fracturado de los cinco instalados inicialmente, debido a que los otros fueron destruidos por descuido con maquinaria que eventualmente compactaba los RSU. Los lixiviados tanto de la celda saneada como de celda abierta fluyen a la única fosa del sitio. El TCA carece de infraestructura para el control de ingreso de los RSU, no cuenta con báscula de pesaje y no se compacta ni se cubre con suelo. Además no cuenta con drenes perimetrales que impidan la infiltración de los escurrimientos pluviales a las celdas y se perciben olores desagradables, así como fauna nociva, principalmente perros, roedores e insectos (moscos, moscas, pulgas y escarabajos). Como puede observarse en la tabla 1, el TCA es un sitio que requiere atención de las autoridades, ya que al no existir ningún tipo de control representa un riesgo a la salud para las comunidades aledañas a corto plazo.

\section{CARACTERIZACIÓN DE RSU}

La tabla 2 concentra la caracterización en porcentaje de los RSU de los sitios estudiados. Cabe mencionar que por cuestiones de operación del RESA, la Celda 1 solo se muestreó en temporada de estiaje y con fines comparativos se presenta la caracterización de la Celda 2 realizada por Delgado (2013). Comparando los subproductos orgánicos entre los dos sitios, el TCA tiene una mayor cantidad (58.49\%) que el RESA (50.39\%), lo cual se debe a que se encuentra en un área agrícola y el RESA está cercano a una zona industrial.

De acuerdo con las categorías propuestas por el Modelo Mexicano de Biogás (SCS, 2009), los subproductos de degradación muy rápida (1) del RESA duplican prácticamente $(25.79 \%)$ el valor de los del TCA $(11.66 \%)$; por el contrario, el TCA triplica (21.90\%) los subproductos de degradación moderadamente rápida (2) al RESA (6.85\%). Para los subproductos de degradación moderadamente lenta (3) y subproductos de degradación lenta (4), el TCA presentó mayor porcentaje $(24.93 \%)$ que el RESA $(17.75 \%)$. Las diferencias anteriormente descritas corroboran lo reportado por Zhang et al. (2013) en cuanto al efecto de los factores propios de cada zona, resaltando en este trabajo el efecto de las actividades económicas. De manera particular, la Celda 3 del RESA en temporada de lluvias es la que presentó mayor porcentaje de subproductos orgánicos $(58.74 \%)$, seguida de la Celda 2 $(50.10 \%)$. La Celda 3 tiene diferencias insignificantes en la composición de residuos orgánicos de degradación muy rápida (1) entre las temporadas de estiaje y lluvias, donde el promedio de ambas temporadas $(26.28 \%)$ representa un valor inferior a la media nacional $(37.97 \%)$ y a la media mundial (43.32\%) (SCS, 2009; OCDE, 2014).

El mayor porcentaje de subproductos de degradación moderadamente rápida (2) y de degradación moderadamente lenta (3) lo tiene la Celda 3 con $8.21 \%$ y $18.18 \%$, respectivamente. La mayor cantidad de subproductos $(1.70 \%)$ de degradación lenta (4) y de inorgáni$\cos (56.53 \%)$ la presenta la Celda 1 , que es la celda de mayor antigüedad, lo que confirma que la degradación de su FO tipo (1) ha sido mayor. Como se dijo anteriormente, los factores ambientales y operacionales influyen en la biodegradación de los RSU y la generación de $\mathrm{CH}_{4}$ (Czepiel et al., 2003; Jokela et al., 2002; Madigan et al., 2004).

El alto contenido de humedad en la temporada de lluvias es notorio en ambos sitios (tabla 3). La Celda 
DOI: http://dx.doi.org/10.22201/fi.25940732e.2017.18n2.013

Díaz-Archundia laura Verónica, Buenrostro-Delgado Otoniel, Mañón-Salas María del Consuelo, Hernández-Berriel María del Consuelo

Tabla 2. Caracterización física de RSU (Muestreo temporadas de lluvias 2013 y estiaje 2014)

\begin{tabular}{|c|c|c|c|c|c|c|c|c|c|}
\hline \multirow{3}{*}{$\begin{array}{c}\text { Sitio } \\
\text { Subproducto }\end{array}$} & \multicolumn{5}{|c|}{ RESA (\%) } & \multicolumn{3}{|c|}{ TCA (\%) } & \multirow{3}{*}{$\begin{array}{c}{ }^{2} \text { Sub- } \\
\text { categorías } \\
\text { Modelo } \\
\text { Mexicano de } \\
\text { Biogás }\end{array}$} \\
\hline & \multirow{2}{*}{$\begin{array}{c}\text { Celda } 1 \\
\text { Estiaje } \\
2014\end{array}$} & \multirow{2}{*}{$\begin{array}{c}\text { Celda } 2 \\
{ }^{1} \text { Estiaje } \\
2012\end{array}$} & \multicolumn{2}{|c|}{ Celda 3} & \multirow[b]{2}{*}{$\begin{array}{c}\text { Promedio } \\
\text { General }\end{array}$} & \multicolumn{2}{|c|}{ TCA } & \multirow[b]{2}{*}{$\begin{array}{c}\text { Promedio } \\
\text { General }\end{array}$} & \\
\hline & & & $\begin{array}{l}\text { Lluvias } \\
2013\end{array}$ & $\begin{array}{l}\text { Estiaje } \\
2014\end{array}$ & & $\begin{array}{c}\text { Lluvias } \\
2013\end{array}$ & $\begin{array}{l}\text { Estiaje } \\
2014\end{array}$ & & \\
\hline Comida & 0.98 & 3.7 & 5.47 & 5.11 & 3.82 & 4.36 & 2.34 & 3.35 & (1) \\
\hline Otros orgánicos & 17.89 & 21.9 & 17.48 & 18.33 & 18.90 & 1.68 & 5.80 & 3.74 & (1) \\
\hline $\begin{array}{c}\text { Pañales ( } 20 \% \text { orgánico / } \\
80 \% \text { inorgánico) }\end{array}$ & 2.12 & 3.9 & 3.10 & 3.06 & 3.05 & 7.54 & 1.60 & 4.57 & (1) \\
\hline Papel higiénico & 2.12 & 3.9 & 3.10 & 3.06 & 3.05 & 15.09 & 3.94 & 9.52 & (2) \\
\hline Poda (jardines) & 1.36 & 3.6 & 7.24 & 3.02 & 3.81 & 1.95 & 22.80 & 12.38 & (2) \\
\hline Textiles & 5.70 & 2.8 & 4.54 & 4.47 & 4.38 & 2.04 & 7.35 & 4.70 & (3) \\
\hline Papel y cartón & 11.58 & 9.2 & 16.79 & 10.56 & 12.03 & 9.39 & 12.43 & 10.91 & (3) \\
\hline Madera & 1.61 & 0.7 & 0.75 & 0.81 & 0.97 & 12.34 & 2.28 & 7.31 & (4) \\
\hline Caucho, piel, huesos y paja & 0.09 & 0.3 & 0.27 & 0.83 & 0.37 & 2.17 & 1.85 & 2.01 & (4) \\
\hline Metales & 1.32 & 1.8 & 0.96 & 1.64 & 1.43 & 1.09 & 3.09 & 2.09 & (i) \\
\hline Construcción y demolición & 4.86 & 6.1 & 1.28 & 4.86 & 4.28 & 1.80 & 2.66 & 2.23 & (i) \\
\hline Vidrio y cerámica & 2.46 & 4.7 & 2.59 & 3.21 & 3.24 & 4.17 & 2.79 & 3.48 & (i) \\
\hline Plásticos & 46.33 & 15.2 & 27.55 & 27.60 & 29.17 & 25.89 & 26.49 & 26.19 & (i) \\
\hline Otros inorgánicos & 1.56 & 22.1 & 8.89 & 13.37 & 11.48 & 10.48 & 4.56 & 7.52 & (i) \\
\hline
\end{tabular}

${ }^{1}$ Delgado, (2013); ${ }^{2}$ SCS, 2009

Orgánicos: 1) Degradación muy rápida: Comida, otros orgánicos (material orgánico difícil de ser identificado por su tamaño y textura) y $20 \%$ de pañales; 2) Moderadamente rápida (poda y papel higiénico); 3) Moderadamente lenta (papel y cartón y textiles), 4) Degradación lenta (madera, caucho, piel, huesos y paja).

Inorgánicos: 1) Metales, $80 \%$ de pañales, construcción y demolición, vidrio y cerámica, Plástico y otros inorgánicos (cabello, piedras y cenizas).

abierta del TCA presenta un valor de humedad atípico (2.53\%) en comparación con los encontrados en temporada de lluvias en el mismo sitio y en el RESA, además de los reportados por Zhu et al., (2009) que oscilan entre $40 \% \mathrm{Hbh}$ y $55 \% \mathrm{Hbh}$, lo anterior se debe a que este sitio lleva diez meses que no recibe RSU y los ahí vertidos no se han removido, cubierto ni compactado.
Los valores de $\mathrm{pH}$, SVT y Hbh que se determinaron en el RESA son similares en las Celdas 1 y 3 , sin embargo en la Celda 2 se obtuvo un $\mathrm{pH}$ básico y menor cantidad de SVT, así como de MO (tabla 3), lo que se atribuye al tipo de residuos recibidos en esa temporada, ya que esta celda recibió más porcentaje de otros inorgánicos (tabla 2).

Tabla 3. Caracterización fisicoquímica de RSU

\begin{tabular}{|c|c|c|c|c|c|c|}
\hline \multirow{3}{*}{$\begin{array}{c}\text { Sitio } \\
\text { Parámetro }\end{array}$} & \multicolumn{4}{|c|}{ RESA } & \multirow{2}{*}{\multicolumn{2}{|c|}{$\frac{\text { TCA }}{\text { Celda abierta }}$}} \\
\hline & \multirow{2}{*}{$\begin{array}{c}\text { Celda } 1 \\
\text { Estiaje } \\
2014\end{array}$} & \multirow{2}{*}{$\begin{array}{c}\text { Celda } 2^{1} \\
\text { Estiaje } \\
2012\end{array}$} & \multicolumn{2}{|c|}{ Celda 3} & & \\
\hline & & & $\begin{array}{l}\text { Lluvias } \\
2013\end{array}$ & $\begin{array}{l}\text { Estiaje } \\
2014\end{array}$ & Lluvias 2013 & $\begin{array}{l}\text { Estiaje } \\
2014\end{array}$ \\
\hline $\mathrm{pH}$ & $7.80 \pm 1.11$ & $8.94 \pm 0.16$ & $7.50 \pm 0.09$ & $6.77 \pm 0.07$ & $7.90 \pm 0.05$ & $7.34 \pm 0.07$ \\
\hline SVT (\%) & $54.53 \pm 8.25$ & $46.18+17.37$ & $58.68 \pm 11.47$ & $56.50 \pm 5.55$ & $84.97 \pm 4.03$ & $60.09 \pm 6.16$ \\
\hline $\mathrm{Hbh}(\%)$ & $29.40 \pm 8.04$ & $25.29 \pm 0.16$ & $38.74 \pm 1.09$ & $15.35 \pm 2.19$ & $40.99 \pm 3.12$ & $2.53 \pm 0.16$ \\
\hline $\mathrm{N}(\%)$ & ND & $0.78 \pm .0 .012$ & $0.85 \pm 0.019$ & $0.68 \pm 0.016$ & $0.90 \pm 0.021$ & $0.58 \pm 0.013$ \\
\hline $\mathrm{C} / \mathrm{N}$ & ND & 14.52 & 15.35 & 28.18 & 24.80 & 25.70 \\
\hline $\begin{array}{c}\text { MO } \\
\text { facilmente oxidable }(\%)\end{array}$ & ND & $19.56 \pm 1.37$ & $22.50 \pm 1.21$ & $33.04 \pm 1.03$ & $38.49 \pm 1.08$ & $35.7 \pm 1.05$ \\
\hline
\end{tabular}

ND. No determinado, ${ }^{1}$ Delgado, (2013) 


\section{Generación de $\mathrm{CH}_{4}$ en pozos de venteo}

En promedio, en ambos sitios se detectó una emisión mayor de $\mathrm{CH}_{4}$ en pozos que en superficie, sin embargo cada celda tiene particularidades.

En la tabla 4 se muestran los promedios de generación en pozos de cada celda. El máximo valor en el RESA supera $50 \%$ v/v de $\mathrm{CH}_{4}$ en varios de sus pozos, valor característico de la biodegradación metanogénica acelerada (Benson et al., 2007; Mor et al., 2006), sin embargo en promedio cada celda presenta un valor inferior a $40 \% \mathrm{v} / \mathrm{v}$ con excepción de la Celda $1(42.21 \% \mathrm{v} / \mathrm{v})$ en temporada de lluvias. Las lecturas obtenidas evidencian que la infiltración de lixiviados y agua de lluvia hacia los RSU a través de la cobertura final y la cantidad de $\mathrm{MO}$, hacen que se incremente significativamente la biodegradabilidad (Mohammed et al., 2009; Zhang et al, 2013). En el resto de pozos, las lecturas de $\mathrm{CH}_{4}$ van desde $5 \% \mathrm{v} / \mathrm{v}$ hasta $25 \% \mathrm{v} / \mathrm{v}$, lo que revela una biodegradación no homogénea, resultado de una menor infiltración en ciertas zonas de los lixiviados recirculados mediante riego en la superficie de las celdas, ya que se detectaron en algunos pozos obstrucciones por lixiviados (taponeamiento).

La Celda saneada del RESA en temporada de lluvias presentó una generación menor de $\mathrm{CH}_{4}$ que en estiaje (tabla 4), que puede deberse a la compactación de las partículas del suelo y a la obstrucción de los poros en la superficie por el agua que recibe, de tal manera que se limita su infiltración. Aunado a lo anterior, esta celda tiene una altura de aproximadamente $20 \mathrm{~m}$ y las
Celdas 2 y 3 están sobrepuestas en parte de ella. Las Celdas 1 y 2 mostraron un comportamiento acorde con las temporadas muestreadas y a la composición de los RSU ahí dispuestos, determinándose mayor generación de $\mathrm{CH}_{4}$ en lluvias que en estiaje.

En la Celda 3 el muestreo y caracterización de biogás en temporada de lluvias se realizó durante su operación (disposición continua de RSU), detectándose una menor generación que en estiaje (tabla 4), lo que puede atribuirse a que en lluvias la emisión de $\mathrm{CH}_{4}$ se dispersó por los alrededores de la celda, mientras que en estiaje las mediciones se hicieron con esta celda ya cubierta con suelo, lo que ayudó a dirigir el biogás a los pozos, contabilizando así una mayor emisión de $\mathrm{CH}_{4}$.

En promedio, la generación en pozos de venteo en temporada de lluvias fue de $579.90 \mathrm{E}-7 \mathrm{~kg} / \mathrm{m}^{2} \mathrm{~h}$ para el RESA y $71.60 \mathrm{E}-7 \mathrm{~kg} / \mathrm{m}^{2} \mathrm{~h}$ para el TCA (tabla 5). Durante estiaje, los niveles fueron $668.23 \mathrm{E}-7 \mathrm{~kg} / \mathrm{m}^{2} \mathrm{~h}$ y $8.18 \mathrm{E}-7$ $\mathrm{kg} / \mathrm{m}^{2} \mathrm{~h}$, respectivamente. En lo que respecta al TCA, los resultados de $\mathrm{CH}_{4}$ corroboran que este sitio es más joven que el RESA, sin embargo, la baja generación detectada de $\mathrm{CH}_{4}$ del único pozo puede ser consecuencia de la fauna nociva (perros y ratas) que requieren de alimento, lo que reduce la MO en los RSU y permite la infiltración del aire.

\section{Generación de Metano en SUPERFICIE}

Las cámaras en el RESA se instalaron principalmente sobre la corona y los caminos de las celdas y en el TCA se ubicaron en la parte poniente del sitio sobre los RSU

Tabla 4. Emisión de $\mathrm{CH}_{4}$ en pozos y superficie durante temporadas de lluvias y estiaje

\begin{tabular}{|c|c|c|c|c|c|c|c|c|}
\hline \multirow{3}{*}{$\begin{array}{c}\text { Sitio de } \\
\text { disposición }\end{array}$} & \multirow{3}{*}{ Celda } & \multirow{3}{*}{$\begin{array}{l}\text { RSU } \\
(\mathrm{t})\end{array}$} & \multicolumn{3}{|c|}{ Temporada de lluvias, 2013} & \multicolumn{3}{|c|}{ Temporada de estiaje, 2014} \\
\hline & & & \multicolumn{2}{|c|}{ Pozos } & \multirow{2}{*}{$\begin{array}{c}\text { Superficie } \\
\left(\mathrm{kgCH}_{4} / \mathrm{m}^{2} \mathrm{~h}\right)\end{array}$} & \multicolumn{2}{|c|}{ Pozos } & \multirow{2}{*}{$\begin{array}{l}\text { Superficie } \\
\left(\mathrm{kgCH}_{4} / \mathrm{m}^{2} h\right)\end{array}$} \\
\hline & & & $(\% \mathrm{v} / \mathrm{v})$ & $\left(\mathrm{kgCH}_{4} / \mathrm{m}^{2} \mathrm{~h}\right)$ & & $(\% \mathrm{v} / \mathrm{v})$ & $\left(\mathrm{kgCH}_{4} / \mathrm{m}^{2} \mathrm{~h}\right)$ & \\
\hline \multirow{4}{*}{ RESA } & Saneada & 545,500 & 16.68 & 2.27 & ND & 28.00 & 14.73 & $80.0 \mathrm{E}-7$ \\
\hline & 1 & 250,000 & 42.21 & 54.09 & $18.0 \mathrm{E}-7$ & 36.12 & 51.58 & $0.02 \mathrm{E}-7$ \\
\hline & 2 & 290,000 & 30.30 & 25.04 & ND & 21.82 & 16.60 & 79.00E-7 \\
\hline & 3 & 480,000 & 12.71 & 9.38 & ND & 31.19 & 24.83 & 13.00E-7 \\
\hline \multirow{2}{*}{ TCA } & Saneada & NR & PI & PI & NC & PI & PI & $\mathrm{NC}$ \\
\hline & Abierta & 394,200 & 15.00 & 2.82 & $2.00 \mathrm{E}-7$ & 6.70 & 0.32 & $0.16 \mathrm{E}-7$ \\
\hline
\end{tabular}

ND: No Determinado; NR: No Reportado; NC: No Cuantificado; PI: Pozos Inhabilitados

Tabla 5. Generación de $\mathrm{CH}_{4}$ por tonelada de RSU (promedio pesado)

\begin{tabular}{ccccc}
\hline \multirow{2}{*}{$\begin{array}{c}\text { Sitio de } \\
\text { disposición }\end{array}$} & \multicolumn{2}{c}{ Temporada de lluvias, 2013} & \multicolumn{2}{c}{ Temporada de estiaje, 2014 } \\
\cline { 2 - 5 } & Pozos & Superficie & Pozos & Superficie \\
& $\mathrm{kg} \mathrm{CH}_{4} / \mathrm{m}^{2} \mathrm{~h} \mathrm{t}$ RSU & $\mathrm{kg} \mathrm{CH}_{4} / \mathrm{m}^{2} \mathrm{~h} \mathrm{t} \mathrm{RSU}$ & $\mathrm{kg} \mathrm{CH}_{4} / \mathrm{m}^{2} \mathrm{~h} \mathrm{t} \mathrm{RSU}$ & $\mathrm{kg} \mathrm{CH}_{4} / \mathrm{m}^{2} \mathrm{~h} \mathrm{t} \mathrm{RSU}$ \\
\hline RESA & $579.90 \mathrm{E}-7$ & $7.08 \mathrm{E}-10$ & $668.23 \mathrm{E}-7$ & $11.00 \mathrm{E}-10$ \\
TCA & $71.60 \mathrm{E}-7$ & $0.42 \mathrm{E}-10$ & $8.18 \mathrm{E}-7$ & $0.040 \mathrm{E}-10$ \\
\hline
\end{tabular}


y sobre la superficie de la Celda saneada (figura 2). Las bajas emisiones detectadas, pueden atribuirse a la compactación de los RSU y a que el suelo de cobertura en las celdas cumple su función (SEMARNAT, 2009). Tomando en cuenta los promedios de las emisiones en superficie y en pozos de venteo por tonelada de RSU, en el RESA fue menor en temporada de lluvias que en estiaje, ya que en esta última temporada fue mayor la recirculación de lixiviados y, por consecuencia, se incrementó la humedad y la MO (Benson et al., 2007; Wrefor et al., 2000).

Cabe mencionar que en el año 2013 se registró una alta precipitación pluvial en ciertas partes del país, dentro de estas se encuentra el municipio donde está ubicado el RESA, lo que provocó erosión en un costado de la Celda saneada y Celda 2. Esto también se debió a que el ángulo del talud es de 2:1, superior a lo recomendado por la ISWA (Laner et al., 2009; Wang et al., 2006). Resultado de lo anterior originó una grieta, que se extendió sobre $60 \mathrm{~m}$ desde la parte superior de la Celda 3 hacia abajo, llegando a la superficie de la Celda saneada. A lo largo de esta grieta se hicieron mediciones con tres campanas, cuantificando en promedio una emisión de $23.7 \mathrm{E}-6 \mathrm{~kg} / \mathrm{m}^{2} \mathrm{~h}$, que es 13 veces superior a la emisión superficial detectada. De aquí la importancia del mantenimiento que debe darse al material de cobertura de las celdas, para evitar que las emisiones fugitivas contribuyan con valores altos de GEI.

En la tabla 5 se muestra el promedio pesado de la generación de los dos sitios en el periodo de muestreo. Respecto a las emisiones en superficie, en la temporada de lluvias fue de $7.08 \mathrm{E}-10 \mathrm{~kg} / \mathrm{m}^{2} \mathrm{~h}$ y $0.42 \mathrm{E}-10 \mathrm{~kg} /$ $\mathrm{m}^{2} \mathrm{~h}$ para el RESA y TCA, respectivamente y en estiaje fue de $11.00 \mathrm{E}-10 \mathrm{~kg} / \mathrm{m}^{2} \mathrm{~h}$ y $0.040 \mathrm{E}-10 \mathrm{~kg} / \mathrm{m}^{2} \mathrm{~h}$, respectivamente.

Las bajas emisiones detectadas superficialmente en el TCA son indicio de su dispersión por los alrededores, ya que la mayoría de los RSU no tienen cobertura intermedia y final. Estas emisiones se pueden considerar insignificantes en comparación con los valores que reporta Chiemchaisri \& Visuanathan (2008), de 8.33E-4 a 3.91 $\mathrm{gCH}_{4} / \mathrm{m}^{2} \mathrm{~h}$; sin embargo, no debe perderse de vista que en México $87 \%$ del total de los sitios registrados son sitios que no tienen control total o son TCA y como lo muestra la concentración detectada en pozos, existe degradación anaeróbica, ya que las concentraciones del $\mathrm{CH}_{4}$ van de 6 a $15 \%$ v/v (tabla 4 ) y a que se reporta que los índices de explosividad van de 4.5 a 14.5\% (PEMEX, 2016).

Con respecto a la Celda saneada del TCA, las emisiones fueron nulas, lo que puede atribuirse a la edad de la celda que excede los 29 años, además del suelo de cobertura compactado y que actualmente en la corona existen montículos de teyolote que cubren el total de la superficie.

\section{Conclusiones}

La aplicación de la LV ayudó a detectar áreas de oportunidad en el RESA. En el TCA la LV resaltó la necesidad de que las autoridades responsables de este sitio tomen acciones para reducir el impacto al medio ambiente y a las comunidades aledañas, para disminuir el pasivo ambiental y promuevan el manejo adecuado de RSU para incrementar el reciclaje de residuos valorizables.

Las diferentes composiciones de RSU en las celdas del RESA son muestra de la variación que puede existir en un mismo SDF. La variabilidad en la composición determinada entre los sitios de la misma zona geográfica de este estudio se atribuye a las actividades particulares de la región en la que está localizado cada sitio. Lo anterior, resalta la importancia de realizar caracterizaciones periódicas a los RSU, con el fin de obtener estimaciones con menor grado de incertidumbre.

Las caracterizaciones realizadas muestran proporciones interesantes de materiales susceptibles a ser recuperados para su reciclaje, con lo que se reduciría sobre $40 \%$ de los RSU dispuestos.

La generación y las emisiones detectadas de $\mathrm{CH}_{4}$ se mostraron influidas por las temporadas estacionales y por las particularidades de cada celda (antigüedad y composición de los RSU, condiciones de operación, recirculación de lixiviados, entre otras). De las emisiones superficiales, el RESA tiene en promedio 7.08E-10 $\mathrm{kg} / \mathrm{m}^{2} \mathrm{~h}$, lo cual puede considerarse despreciable comparado con la generación en pozos, destacando que se debe evitar la existencia de grietas, ya que podrían elevar las emisiones más de 13 veces a las detectadas en superficie.

En el TCA fueron mayores las emisiones superficiales en temporada de lluvias, las cuales a pesar de presentar valores pequeños, muestran la necesidad de implementar un programa de saneamiento y seguimiento del sitio.

El monitoreo del biogás permitió tener mayor certeza de la contribución de GEI de los sitios de disposición estudiados, lo cual es relevante para mejorar su operación, contemplar su aprovechamiento y reducir su impacto al ambiente.

\section{Agradecimientos}

Los autores agradecen al Instituto Tecnológico de Toluca, al Consejo Nacional de Ciencia y Tecnología, a la 
empresa Mantenimiento y Servicios Ambientales (MASERA) y a la Universidad Michoacana de San Nicolás de Hidalgo por el apoyo brindado para el desarrollo del presente estudio.

\section{RefERENCIAS}

ADEME. Agence de l'Environment et de la Maltrise de l'Energie, field comparision of methods for landfill fugitive methane emissions measurement, Convention ADEME, 2008, 07-74C0034.

APHA. Standard methods for water and wastewater examination, 21st ed., American Public Health Association-American, Water Works Association, Water Environment Federation Publication, Washington, 2005.

Babilotte A., Lagier T., Fiani E., Taramini V. Fugitive methane emissions from landfills: field comparision of five methods on a french landfill. Journal of Environmental Engineering@ASCE, 2010: 777-784.

Barry D., Gregory B., Harries C. Minimizing methane emissions from MSW landfill, Atkins Environment, UK, 2004.

Benson C.H., Barlaz M.A., Lane D.T., Rawe J.M., Practice review of five bioreactor/recirculation landfills. Waste Manage, volumen 27, 2007: 13-29.

Chen Y., Cheng Jay J., Creamer-Kurt S. Inhibition of anaerobic digestion process: A review. Bioresource Techonology, volumen 99, 2008: 4044-4064.

Chiemchaisri C., Visvanathan C. Greenhouse gas emission potential of the municipal solid waste disposal sites in Thailand. Journal of the Air and Waste Management Association, volumen 58, 2008: 628-635.

CICC. Secretaría de Medio Ambiente y Recursos Naturales, Comisión Intersecretarial de Cambio Climático, hacia una estrategia nacional de acción climática, SEMARNAT, México, 2006.

Czepiel P., Shorter J., Mosher B., Allwine E., McManus J., Harriss R., Kolb C., Lamb B. The influence of atmospheric pressure on landfill methane emissions. Waste Manage, volumen 23 (número 7), 2003: 593-598.

Delgado-Hernández C. Estudio de parámetros fisicoquímicos en lixiviados recirculados en residuos sólidos urbanos (tesis de maestría), Instituto Tecnológico de Toluca, Metepec, Estado de México, agosto 2013.

EA. Environment Agency. How to comply with your environmental permit additional guidance for: Landfill (EPR 5.02), GEHO0209BP JD-E-E, 2009.

EPA. Environmental Protection Agency, Landfill Manuals, Landfill Site Design, Ireland, 2000.

GMI. Global methane initiative, solid waste disposal site design and operational considerations, Chapter 2, International best practices guide for LFGE projects-2012.

Hyung-Kim M., Byul-Song H., Song Y., Jeong T., Kim-Jung W.K. Evaluation of food waste disposal options in terms of global warming and energy recovery: Korea. International Journal of Energy and Environmental Engineering, volumen 4, 2013:1.

INE. Instituto Nacional de Ecología, Potencial de mitigación de GEI en México al 2020 en el contexto de la cooperación internacional, México, 2010.

INECC. Instituto Nacional de Ecología y Cambio Climático, quinta comunicación nacional ante la convención marco de las naciones unidas sobre el cambio climático, México, 2012 [en línea]. Disponible en: http:==www2:ine:gob:mx=publicacione $\mathrm{s}=$ new:consultaPublicacion:php.

INEGI. Instituto Nacional de Estadística y Geografía, Estadísticas Nacionales, México, 2016 [en línea]. Disponible en: http://cuentame.inegi.org.mx/poblacion/default.aspx?tema=P, Población.

IERMRSUALC. Informe de la evaluación regional del manejo de residuos sólidos urbanos en ALC 2010, IDB-MG-115; 2010, AIDIS-001/2011.

Jokela J.P.Y., Kettunen R.H., Rintala J.A. Methane and leachate pollutant emission potential from various fractions of $\mathrm{mu}-$ nicipal solid waste (MSW): Effects of source separation and aerobic treatment. Waste Manage Res, volumen 20, 2002:424433.

Laner D., Fellner J., Brunner P.H., Flooding of municipal solid waste landfills-An environmental hazard? Sci Total Environ, volumen 407, 2009: 3674-3680.

LANDTEC 2009. GEM2000 Instruments operation manual, gas analyzer \& extraction monitors. www.LANDTECNA.com

López-Martínez A. Cuantificación de emisiones difusas mediante cámara de flujo en un vertedero de residuos urbanos, Universidad de Valladolid, REDISA (Red de ingeniería de saneamiento ambiental), ISBN: 978-85-7745-589-8, 2010, Joao Pessoa- PB, Brasil, 08 a 19 de Septiembre.

Madigan M.T., Martinko J.M., Parker J.B. Biología de los microorganismos, 10 ed., Pearson Educación S.A., España, 2004.

Mohammed F.M.A., Noor E.A.B., Abdul A.H.K. Review on landfill gas emission to the atmosphere. European Journal of Scientific Research, volumen 30 (número 3), 2009: 427-436.

Mor S., Ravindra K., De Visscher A., Dahiya R.P., Chandra A. Municipal solid waste characterization and its assessment for potential methane generation: A case study. Sci Total Environ, volumen 371, 2006: 1-10.

OCDE. Organization for Economic Co-operation and Development, 2014a [en línea]. Disponible en: http://www.oecd.org/

OCDE-1. Organization for Economic Co-operation and Development, 2014b, Country Reviews, 25-06-2014 [en línea]. Disponible en: http://www.oecd.org/env/country-reviews/.

PEMEX. Hoja de seguridad para sustancias químicas, PEMEX, gas y petroquímica básica, 2016.

SCS Engineers. Manual del usuario modelo mexicano de biogás versión 2.0. SCS Engineers Phoenix, AZ 85008. Contrato de EPA No. EP - W -06-023, 2009. Actividad 30. Landfill Methane Outreach Program. Agencia para la Protección del Ambiente (U.S. EPA). 
SEMARNAT. Secretaría del Medio Ambiente y Recursos Naturales, 2009, Manual de especificaciones técnicas para la construcción de rellenos sanitarios para Residuos Sólidos urbanos (RSU) y Residuos de Manejo Especial (RME), Dirección de Manejo Sustentable de Residuos Sólidos, Contrato DGRMISDCA-DGFAUT-No. 018/2009.

SEMARNAT-1. Secretaría del Medio Ambiente y Recursos Naturales, Marco Jurídico del Sector Medio Ambiente, 2016, México [en línea]. Disponible en: http:// www. semarnat. gob.mx/ leyesynormas/Pages /nmx-residuos.aspx.

SEMARNAT-INECC. 2012. Diagnóstico básico para la gestión integral de los residuos.

Spokas K., Graff C., Morcet M., Aran C. Implications of spatial variability of landfill emission rates on geospatial analyses. Waste Manage, volumen 23, 2003: 599-607.

Wang-Yao K., Towprayoon S., Chiemchaisri Ch., Gheewala Sh.H., Nopharatana A. Seasonal variation of landfill methane emissions from seven solid waste disposal sites in central Thailand, 2nd Joint International Conference on Sustainable Energy and Environment (SEE2006), 2006, Bangkok, Thailand.

Wrefor K.A., Atwater J.W., Lavkulich L.M. The efects of moisture imputs on landfill gas production and composition and leachate characteristics at the Vancouver Landfill Site at Burns Bog. Waste Manage Res, volumen 18, 2000: 386-392.

Zhang H., Pinjing H., Liming S. Methane emissions from MSW landfill with sandy soil covers under leachate recirculation and subsurface irrigation. Atmospheric Environment, volumen 42, 2008: 5579-5588.

Zhang H., Yan X., Cai Z., Zhang Y. Effect of rain fall on the diurnal variations of $\mathrm{CH}_{4}, \mathrm{CO}_{2}$, and $\mathrm{N}_{2} \mathrm{O}$ fluxes from municipal solid waste landfill. Sci Total Environ, volumen 442, 2013: 73-76.

Zhu B., Gikas P., Zhang R., Lord J., Jenkins B., Li X. Characteristics and biogas production potential of municipal solid wastes pretreated with a rotary frum reactor. Bioresource Technology, volumen 100, 2009: 1122-1129.

\section{Citación sugerida:}

\section{Citación estilo Chicago}

Díaz-Archundia, Laura Verónica, Otoniel Buenrostro-Delgado, María del Consuelo Mañón-Salas, María del Consuelo Hernández-Berriel. Emisión de gases de efecto invernadero de residuos sólidos urbanos en dos diferentes sitios de disposición final en México. Ingeniería Investigación y Tecnología, XVIII, 02 (2017): 149-159.

\section{Citación estilo ISO 690}

Díaz-Archundia L.V., Buenrostro-Delgado O., Mañón-Salas M. del C., Hernández-Berriel $\mathrm{M}$ del $\mathrm{C}$. Emisión de gases de efecto invernadero de residuos sólidos urbanos en dos diferentes sitios de disposición final en México. Ingeniería Investigación y Tecnología, volumen XVIII (número 2), abril-junio 2017: 149-159.

\section{Semblanzas de los autores}

Laura Verónica Díaz-Archundia. Estudiante de doctorado en el Instituto Tecnológico de Toluca con la tesis sobre el diagnóstico de gas metano en SDF. Obtuvo su maestría en la Universidad Autónoma de Querétaro con la tesis: “Tratamiento de residuos mixtos con plasma térmico" en el Instituto Nacional de Investigaciones Nucleares. Trabajó en el Departamento de medio ambiente y auditorías en el mismo Instituto.

Otoniel Buenrostro-Delgado. Es investigador en el Instituto de Investigación de Agronomía y Forestal (IIAF), Universidad Michoacana de San Nicolás de Hidalgo. Sus campos de investigación son: la contaminación y el tratamiento de residuos sólidos, planificación de sistemas de gestión de residuos sólidos y emisiones de gases de efecto invernadero en los SDF.

María del Consuelo Mañón-Salas. Obtuvo su doctorado en la Universidad Autónoma de Baja California, en el ámbito del medio ambiente. Desarrolló modelos para estimar los lixiviados y biogás a partir de RSU en el proceso de biodegradación, herramientas softcomputing anaeróbicas. Actualmente realiza un trabajo posdoctoral titulado: El desarrollo de un sistema multiagente para la simulación del proceso de compostaje de residuos sólidos orgánicos y biosólidos.

María del Consuelo Hernández-Berriel. Estudió el doctorado en ciencias biológicas en la Universidad Autónoma de San Nicolás de Hidalgo. Es profesora de investigación a tiempo completo en la maestría en ciencias en ingeniería ambiental y en el doctorado en ciencias ambientales del Instituto Tecnológico de Toluca. Su campo de investigación incluye: gestión ambiental y tratamiento de residuos sólidos. Es miembro de la Sociedad Mexicana de la Ciencia y Tecnología Aplicada y Residuos Sólidos (SOMERS) y miembro de la Red Iberoamericana en Gestión y Aprovechamiento de Residuos (REDISA). 